Pacific Journal of Mathematic 


\title{
A SIMPLE MEASURE-PRESERVING TRANSFORMATION WITH TRIVIAL CENTRALIZER
}

\author{
ANDRÉS DEL JUNCO
}

\begin{abstract}
We show that a certain simple and well-known measurepreserving transformation due to Chacón has the property of commuting only with its powers. We also state a theorem concerning the centralizer of certain rank one transformations. In addition we state without proof the fact that Chacón's example has no factors.
\end{abstract}

In [5] Ornstein showed that there exists an invertible measurepreserving transformation (automorphism) which is mixing and rank one and, moreover, that any such automorphism has trivial centralizer, i.e., commutes only with its powers. The purpose of this note is to show that a simple and well-known example of a weakly-mixing automorphism, which is due to Chacón [3], also has trivial centralizer (Theorem 1). It is worth observing that Theorem 1 is false for an arbitrary weakly-mixing rank one automorphism. In fact, it is possible to construct a weakly-mixing rank one automorphism $S$ which splits, $S=S_{1} \times S_{2}$.

Although the mixing property of Ornstein's example makes it more remarkable there are at least two reasons why Chacón's example is of some interest in this context. First Ornstein's construction is complicated and nonexplicit in nature, as opposed to Chacón's which could hardly be simpler or more explicit. Secondly, even the examples of weakly-mixing transformations having no roots constructed in $[2,1]$ are quite difficult compared with the present one which actually does much more.

Chacón's transformation can be most efficiently described as follows. Let $T$ be the shift on $\{0,1\}^{z}$ and define by recursion the finite sequences $\sigma(n)$ of 0 's and 1's:

$$
\begin{gathered}
\sigma(0)=0 \\
\sigma(n)=\sigma(n-1) \sigma(n-1) 1 \sigma(n-1) . \\
(\sigma(1)=0010, \sigma(2)=0010001010010, \text { etc. })
\end{gathered}
$$

Let $\overline{O^{\circ}} \subset\{0,1\}^{Z}$ consist of those sequences $x$ such that each finite segment of $x$ is a segment of $\sigma(n)$ for some $n$. Then it is easy to show that $(\overline{\mathcal{O}}, T)$ is a minimal uniquely ergodic system so there is a unique $T$-invariant Borel measure $\mu$ on $\{0,1\}^{z}$ which is supported on $\overline{\mathcal{O}}$. $\mu$ can be defined explicitly on finite sequences $x$ (that is, on cylinder sets) by 


$$
\mu(x)=\lim _{n \rightarrow \infty} f r(x, \sigma(n))
$$

where $f r(x, \sigma(n))$ denotes the frequency of appearance of $x$ as a segment of $\sigma(n)$.

To see that $T$ is (isomorphic to) Chacón's example one takes in his geometric construction the partition $\left\{P_{0}, P_{1}\right\}$ where $P_{0}$ consists of the initial single level of the construction and $P_{1}$ all levels added later on. $P$ is a generator (this follows from Lemma 2 below) and hence sets up an isomorphism of the geometric construction with $T$. It is easily seen that the image measure is supported on $\bar{\sigma}$ and satisfies (1).

We can now state the main result.

THEOREM 1. If $\varphi$ is a (not necessarily invertible) measure-preserving transformation of $\left(\{0,1\}^{z}, \mu\right)$ and $\phi T=T \varphi$ then $\varphi=T^{k}$ for some integer $k$.

For the proof we need a bit of terminology and three lemmas. If $\alpha$ is a sequence of 0 's and 1's of length $n$ we write $|\alpha|=n$. For $i, j \in Z[i, j]=\{k \in Z: i \leqq k \leqq j\}$ and if $x \in\{0,1\}^{Z}$ we write $x[i, j]$ for the segment of $x$ of length $j-i+1$ beginning at $i$ and ending at $j$. If $x[i, j]=\alpha$ we say $\alpha$ occurs at $i$ in $x$. For $x$ and $y$ finite sequences of length $n, \bar{d}(x, y)=1 / n \#\{i: x(i) \neq y(i)\}$. (We think of a finite sequence as a string of symbols with no specified index set but for definitions like the above $x$ and $y$ must be indexed in an obvious way.) For $(x, y) \in\{0,1\}^{z}, \bar{d}(x, y)=\lim _{i, j \rightarrow \infty} \bar{d}(x[-i, j], y[-i, j])$ if the limit exists.

Any measurable transformation $\varphi$ of $\left(\{0,1\}^{Z}, \mu\right)$ which commutes with the shift is called a code. $\varphi$ is said to be finite if there is an $n$ such that $(\varphi x)(0)$ depends only on $x_{-n}^{n}$ for a.a.x. In this case we write $|\varphi| \leqq n$. Any code $\phi$ can be approximated by a finite code in the sense that for each $\varepsilon>0$ there is a finite code $\varphi^{\prime}$ such that $\bar{d}\left(\varphi(x), \varphi^{\prime}(x)\right)<\varepsilon$ for a.a.x. (Approximate $\varphi^{-1}\left(P_{0}\right)$ by cylinder sets and apply the erodic theorem.) In this case we write $\varphi \stackrel{\varepsilon}{\sim} \varphi^{\prime}$.

For purpose of the following lemma only, we adopt $[0,|\sigma(n)|-1]$ as the index set of $\sigma(n)$ and let $I_{n, m} \subset[0,|\sigma(n+m)|-1]$ be the set of indices corresponding to initial entries of the component $\sigma_{n}$ 's of $\sigma_{n+m}$. The following lemmas say that $\sigma(n)$ occurs in $\sigma(n+m)$ only where we expect it.

Lemma 2. If $\sigma(n)$ occurs at $i$ in $\sigma(n+m)$ then $i \in I_{n, m}$.

Proof. This is easy to see by induction on $n$. 
REMARK. It follows from Lemma 2 that if $x \in \bar{\sigma}$ and $\sigma(n)$ and $\sigma(n+m)$ occur in $x$ on nondisjoint sets then $\sigma(n)$ is contained entirely in $\sigma(n+m)$.

The copies of $\sigma(n)$ which appear in $\sigma(n+1)$ (there are only the three obvious ones, by Lemma 2) will be said to have orders 1,2 , and 3 respectively, counting from the left. Similarily if $x \in \bar{O}$ any $\sigma(n)$ appearing in $x$ has a unique order determined by its position in the $\sigma(n+1)$ containing it.

LEMMA 3. Suppose $\alpha$ occurs at $i$ in $\sigma(n)(n \geqq 5),|\alpha|>\left|\sigma_{n-3}\right|$ and $\beta$ is the string of length $|\alpha|$ which occurs at $i+1$. Then $\bar{d}(\alpha, \beta)>$ $10^{-3}$.

Proof. Let $\sigma(n)^{*}$ (respectively $\sigma(n)_{*}$ ) denote $\sigma(n)$ with the final (respectively initial zero) deleted. Then it is easy to see that for all $n, \bar{d}\left(\sigma(n)^{*}, \sigma(n)_{*}\right)>\bar{d}\left(\sigma(1)^{*}, \sigma(1)_{*}\right)=2 / 3$. Since $|\alpha|>|\sigma(n-3)|$ there must be a $\sigma(n-4)^{*}$ and $\sigma(n-4)_{*}$ occuring in the same relative positions in $\alpha$ and $\beta$ respectively. This forces

$$
\bar{d}(\alpha, \beta)>\frac{\left|\sigma(n-4)^{*}\right|}{|\sigma(n)|} \frac{2}{3}>\frac{1}{4^{4}} \frac{2}{3}>10^{-3} .
$$

Lemma 4. Suppose $\varphi$ is a code and $\varphi^{\prime}$ is a finite code such that $\left|\varphi^{\prime}\right|<10^{-8}|\sigma(N-2)|, N \geqq 5$. Consider an $x \in\{0,1\}^{z}$ such that $\varphi(x) \in \overline{O^{\circ}}$ and suppose that for some $n \geqq N, \sigma(n)$ occurs at $i$ in $x$ and codes under $\varphi^{\prime}$ with error less than $10^{-8}$, that is

$$
\bar{d}\left(\varphi^{\prime}(x)[i, i+|\sigma(n)|-1], \varphi(x)[i, i+|\sigma(n)|-1]\right)<10^{-8} .
$$

Then there is a $k$ such that $|k|<|\sigma(n-2)|$ and $\sigma(n)$ occurs at $i+k$ in $\varphi(x)$.

Proof. Since $\varphi(x) \in \overline{\mathcal{O}}, \sigma(n)$ must occur at some $j$ in $x$ with

$$
i-\frac{|\sigma(n)|}{2}-1 \leqq j \leqq i+\frac{|\sigma(n)|}{2}+1 .
$$

(Since $\varphi(x) \in \overline{O^{3}}$ any $|\sigma(n)|+1$ consecutive indices must contain the beginning of a $\sigma(n)$.) If the lemma is false then either

$$
i+|\sigma(n-2)| \leqq j \leqq i+\frac{|\sigma(n)|}{2}+1
$$




$$
i-\frac{|\sigma(n)|}{2}-1 \leqq j \leqq i-|\sigma(n-2)| \cdot
$$

We now consider these cases separately and arrive at a contradiction in each. In case (2) we have the following picture:

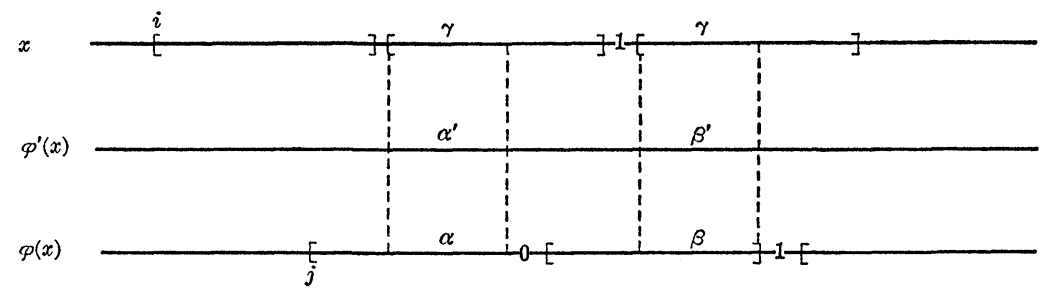

Here square brackets denote beginnings and ends of $\sigma(n-1)$ 's and the indicated zero is a final zero in a $\sigma(n-1)$. We have depicted $j<i+|\sigma(n)|$ but the picture is intended to cover the case $j \geqq i+$ $|\sigma(n)|$ as well. In either case the leftmost $\gamma$ shown in $x$ is $x[m, n]$ where $[m, n]=[i+|\sigma(n)|, i+2|\sigma(n)|-1] \cap[j, j+|\sigma(n)|-2]$. Note that $\#[m, n] \geqq|\sigma(n-2)|-1 \geqq|\sigma(n-3)|$ since we are in case (2).

Now since $\alpha^{\prime}$ and $\beta^{\prime}$ are both coded from $\gamma$ they will agree except for end-effects which are in number less than

$$
2\left|\phi^{\prime}\right|<2 \cdot 10^{-8}|\sigma(N-2)|<10^{-7}|\sigma(n-2)| \text {. }
$$

Thus

$$
\bar{d}\left(\alpha^{\prime}, \beta^{\prime}\right)<10^{-7} \frac{|\sigma(n-2)|}{\left|\alpha^{\prime}\right|}<10^{-7} \frac{|\sigma(n-2)|}{|\sigma(n-3)|}<10^{-6} .
$$

Moreover since $x[i, i+|\sigma(n)|-1]$ codes with error less than $10^{-8}$

$$
\bar{d}\left(\alpha, \alpha^{\prime}\right)<\frac{|\sigma(n)|}{|\alpha|} 10^{-8}<4^{4} 10^{-8}<10^{-5},
$$

and the same for $\bar{d}\left(\beta, \beta^{\prime}\right)$. Thus

$$
\bar{d}(\alpha, \beta)<10^{-6}+10^{-5}+10^{-5}<10^{-3}
$$

which contradicts Lemma 3, since the relative positions of $\alpha$ and $\beta$ in $\sigma(n)$ differ by one.

In case (3) the picture is

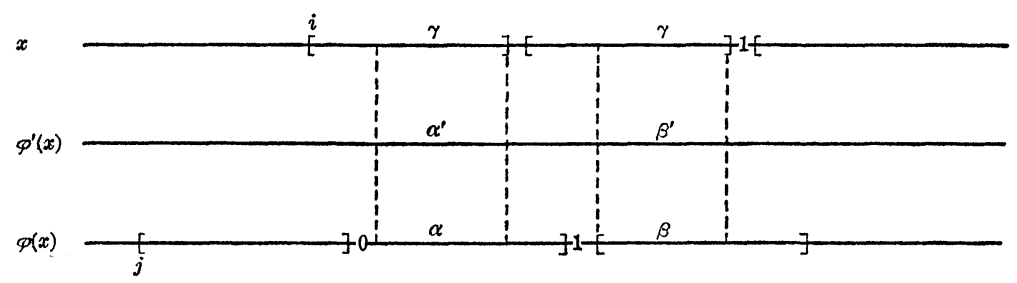


where we have indicated an initial zero of a $\sigma(n-1)$ and we arrive at a contradiction in a similar way.

Proof of Theorem 1. Choose a finite code $\varphi^{\prime}$ such that $\varphi^{\prime} \stackrel{10^{-8}}{\sim} \varphi$. Fix an $x \in \overline{O^{-}}$such that $\varphi(x) \in \overline{O^{\prime}}$ (this is true for a.a.x since $\varphi$ is measure-preserving) and $\bar{d}\left(\varphi(x), \varphi^{\prime}(x)\right)<10^{-8}$ (which is also true for a.a.x). Since $x \in \overline{\mathcal{O}}$ we may choose sequences $i(n) \rightarrow-\infty$ and $j(n) \rightarrow \infty$ such that $x[i(n), j(n)]=\sigma(n)$. Fix $N \geqq 5$ such that $\left|\varphi^{\prime}\right| \leqq 10^{-8}|\sigma(N-2)|$ and

$$
\bar{d}\left(\varphi(x)[i(n), j(n)], \varphi^{\prime}(x)[i(n), j(n)]\right)<10^{-8} \text { for all } n \geqq N .
$$

Now by Lemma $4, \sigma(N)$ occurs at $i(N)+k$ in $\varphi(x)$ for some $|k|<|\sigma(N-2)|$. Applying Lemma 4 again we find that $\sigma(N+1)$ occurs at $i(N+1)+l$ in $\varphi(x)$ for some $|l|<|\sigma(N-1)|$ and this $\sigma(N+1)$ must contain the $\sigma(N)$ appearing at $i(N)+k$ in $\varphi(x)$, since $|\sigma(N-2)|+|\sigma(N-1)|<|\sigma(N)|$ (see the remark following Lemma 1). We claim that the order of the $\sigma(N)$ at $i(N)+k$ in $\varphi(x)$ is the same as that of the $\sigma(N)$ at $i(N)$ in $x$. This is so because otherwise we would have

$$
|l| \geqq|\sigma(N)|-|k| \geqq|\sigma(N)|-|\sigma(N-2)| \geqq 2|\sigma(N-1)|,
$$

a contradiction. It follows that the orders are the same, as claimed, which obviously implies that $l=k$.

Now we apply Lemma 4 a third time and conclude in the same manner that $\sigma(N+2)$ occurs at $i(N+2)+k$. Continuing in this way we find that $\sigma(N+m)$ occurs at $i(N+m)+k$ in $\varphi(x)$ for all $m \geqq 0$. Since $i(n) \rightarrow-\infty$ and $j(n) \rightarrow \infty$ this implies that $\varphi(x)=T^{k} x$. Of course $k$ depends on $x$ but since there is such a $k$ for a.a.x, there is a fixed $k$ such that $\varphi(x)=T^{k} x$ for $x \in E, \mu(E)>0$. It then follows from the ergodicity of $T$ that $\varphi(x)=T^{k} x$ for a.a.x.

We could also have obtained Theorem 1 as a corollary of the following theorem. To state it we first describe (somewhat informally) a certain class of automorphisms to which the $T$ of Theorem 1 belongs. A rank one automorphism (this notion has gone by many names in the past- in [5] it is called class 1) is an automorphism $S$ such that for any partition $P$, the $(P, S)$ names have the following structure: Given $\varepsilon$ almost have every name $\xi$ is within $\varepsilon$ (in $\bar{d}$ ) of a sequence $\eta$ which is a concatenation of copies of a single finite string $\eta_{0}$ and arbitrary "joining" strings such that the joining strings occupy a proportion less than $\varepsilon$ of the places in $\eta$. If $\eta$ can be chosen so that the joining strings have only two lengths $a$ and $b$ and if the number of successive joiners of length $a$ is bounded then we'll say that $S$ is rank one with two return times. For the $T$ of Theorem 1 , the two return times are just 0 and 1. 
THEOREM 5. If $S$ is rank one with two return times then the set of measure-preserving transformations which commute with $S$ is the weak closure [4, p. 61] of the group generated by $S$.

Theorem 1 follows from Theorem 5 as follows. If $\varphi T=T \varphi$ and $T^{k_{i}} \rightarrow \varphi$ weakly for some nondecreasing sequence $k_{i}$ then $T^{k_{i}-k_{i-1}}$ converges weakly to the identity. One then shows, by arguments as in Lemmas 2 and 3 , that for $x \in \overline{\mathcal{O}}, \bar{d}\left(x, T^{k} x\right)>10^{-100}$ for all $k \neq 0$, so that $k_{i}-k_{i-1}=0$ for large $i$, forcing $\varphi=T^{k}$.

We do not know whether Theorem 5 is valid for any rank one $S$.

We conclude by stating without proof another property of $T$. It is known [6] that a mixing rank one automorphism has only the trivial factor algebras. The same holds for the $T$ of Theorem 1:

THEOREM 6. Up to null sets the only $\sigma$-algebras invariant under $T$ are the full $\sigma$-algebra and the trivial $\sigma$-algebra $\{\phi, \bar{O}\}$.

Theorem 6 is proved by coding arguments similar to those in Theorem 1. The idea is that if $\varphi$ is a homomorphism of $T$ which is not $1-1$ we can find $x, y \in \overline{\mathcal{O}}$ such that $x \neq T^{n} y$ for all $n \in Z$ and $\varphi(x)=\varphi(y)$. Approximating $\varphi$ by a finite code $\varphi^{\prime}$ one then shows that $\bar{d}\left(\varphi^{\prime}\left(\sigma(n)^{*}\right), \varphi^{\prime}\left(\sigma(n)_{*}\right)\right.$ ) (notation as in Lemma 3 ) is small for large $n$. One concludes that $\bar{d}(\varphi(x), \varphi(T x))=0$ for $x \in \overline{\mathcal{O}}$, which implies that $\varphi$ is constant by the ergodicity of $T$.

\section{REFERENCES}

1. R. V. ChaCón, A geometric construction of measure preserving transformations, Proc. Fifth Berkeley Symp. Math. Stat. Prob. vol II part 2, 335-360, University of California Press (1967).

2. - Transformations having continuous spectrum, J. Math. and Mechanics, 16 (1969), 399-415.

3. - Weakly mixing transformations which are not strongly mixing, Proc. Amer. Math. Soc., (1969), 559-562.

4. P. R. Halmos, Ergodic Theory, Chelsea, New York (1956).

5. D. S. Ornstein, On the root problem in ergodic theory, Proc. Sixth Berkeley Symp. Math. Stat. Prob. vol II, 347-356, University of California Press (1967).

6. S. Polit, Thesis, Stanford University (1974).

Received December 18, 1977.

The Ohio State University

Columbus, OH 43210 


\section{PACIFIC JOURNAL OF MATHEMATICS}

\section{EDITORS}

RichaRd ARENS (Managing Editor)

University of California

Los Angeles, CA 90024

Charles W. Curtis

University of Oregon

Eugene, OR 97403

C. C. Moore

University of California

Berkeley, CA 94720

\section{J. DUGUNDJI}

Department of Mathematics

University of Southern California

Los Angeles, CA 90007

R. FinN and J. Milgram

Stanford University

Stanford, CA 94305

\section{E. F. BECKENBACH \\ B. H. NeumanN

\author{
UNIVERSITY OF BRITISH COLUMBIA \\ CALIFORNIA INSTITUTE OF TECHNOLOGY \\ UNIVERSITY OF CALIFORNIA \\ MONTANA STATE UNIVERSITY \\ UNIVERSITY OF NEVADA, RENO \\ NEW MEXICO STATE UNIVERSITY \\ OREGON STATE UNIVERSITY \\ UNIVERSITY OF OREGON
}

F. WOLF

K. Yoshida

\section{SUPPORTING INSTITUTIONS}

\author{
UNIVERSITY OF SOUTHERN CALIFORNIA \\ STANFORD UNIVERSITY \\ UNIVERSITY OF HAWAII \\ UNIVERSITY OF TOKYO \\ UNIVERSITY OF UTAH \\ WASHINGTON STATE UNIVERSITY \\ UNIVERSITY OF WASHINGTON
}

The Supporting Institutions listed above contribute to the cost of publication of this Journal, but they are not owners or publishers and have no responsibility for its content or policies.

Mathematical papers intended for publication in the Pacific Journal of Mathematics should be in typed form or offset-reproduced, (not dittoed), double spaced with large margins. Please do not use built up fractions in the text of the manuscript. However, you may use them in the displayed equations. Underline Greek letters in red, German in green, and script in blue. The first paragraph or two must be capable of being used separately as a synopsis of the entire paper. Items of the bibliography should not be cited there unless absolutely necessary, in which case they must be identified by author and journal, rather than by item number. Manuscripts, in triplicate, may be sent to any one of the editors. Please classify according to the scheme of Math. Reviews, Index to Vol. 39. All other communications should be addressed to the managing editor, or Elaine Barth, University of California, Los Angeles, California, 90024.

50 reprints to each author are provided free for each article, only if page charges have been substantially paid. Additional copies may be obtained at cost in multiples of 50 .

The Pacific Journal of Mathematics is issued monthly as of January 1966. Regular subscription rate: $\$ 72.00$ a year (6 Vols., 12 issues). Special rate: $\$ 36.00$ a year to individual members of supporting institutions.

Subscriptions, orders for numbers issued in the last three calendar years, and changes of address should be sent to Pacific Journal of Mathematics, P.O. Box 969, Carmel Valley, CA 93924, U.S.A. Older back numbers obtainable from Kraus Periodicals Co., Route 100, Millwood, NY 10546.

PUBLISHED BY PACIFIC JOURNAL OF MATHEMATICS, A NON-PROFIT CORPORATION

Printed at Kokusai Bunken Insatsusha (International Academic Printing Co., Ltd.). 8-8, 3-chome, Takadanobaba, Shinjuku-ku, Tokyo 160, Japan. 


\section{Pacific Journal of Mathematics \\ Vol. 79, No. $2 \quad$ June, 1978}

David R. Adams, Quasi-additivity and sets of finite $L^{p}$-capacity ........ 283

George M. Bergman and Warren Dicks, Universal derivations and universal

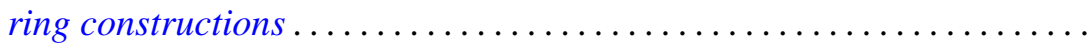

Robert F. Brown, Addendum to: "Fixed points of automorphisms of compact Lie groups".........................................

Eugene Frank Cornelius, Jr., Characterization of a class of torsion free

groups in terms of endomorphisms .......................

Andres del Junco, A simple measure-preserving transformation with trivial centralizer..................................... 357

Allan Lee Edmonds, Extending a branched covering over a handle ...... 363

Sjur Flam, A characterizaton of $\mathbf{R}^{2}$ by the concept of mild convexity .......

Claus Gerhardt, $L^{p}$-estimates for solutions to the instationary Navier-Stokes

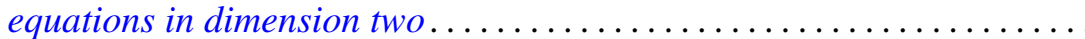

Kensaku Gomi, Finite groups with a standard subgroup isomorphic to

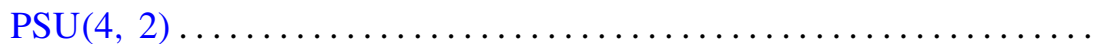

E. E. Guerin, A convolution related to Golomb's root function ........... 463

$\mathrm{H}$. B. Hamilton, Modularity of the congruence lattice of a commutative cancellative semigroup .................................

Stephen J. Haris, Complete reducibility of admissible representations over function fields.

Shigeru Itoh and Wataru Takahashi, The common fixed point theory of singlevalued mappings and multivalued mappings... ...

James E. Joseph, Multifunctions and graphs .............

Bruce Magurn, Images of $S K_{1} Z G$. .

Arnold Koster Pizer, A note on a conjecture of Hecke... .

Marlon C. Rayburn, Maps and h-normal spaces ...........

Barada K. Ray and Billy E. Rhoades, Corrections to: "Fixed-point theorems for mappings with a contractive iterate" ..............

Charles Irvin Vinsonhaler, Corrections to: "Torsion free abelian groups quasiprojective over their endomorphism rings. II". . . 